\title{
HILBERT SPACES OF ANALYTIC FUNCTIONS BETWEEN THE HARDY AND THE DIRICHLET SPACE
}

\author{
ALEXANDRU ALEMAN
}

(Communicated by Paul S. Muhly)

\begin{abstract}
For a large class of Hilbert spaces of analytic functions in the unit disc lying between the Hardy and the Dirichlet space we prove that each element of the space is the quotient of two bounded functions in the same space. It follows that the multiplication operator on these spaces is cellular indecomposable and that each invariant subspace contains nontrivial bounded functions.
\end{abstract}

\section{INTRODUCTION}

For a positive integrable function $w \in C^{2}[0,1)$, consider the space $H_{w}$ of analytic functions $f$ in the unit disc $U$ that satisfies

$$
\|f\|_{w}^{2}=|f(0)|^{2}+\int_{U}\left|f^{\prime}(z)\right|^{2} w(|z|) d m<\infty,
$$

where $m$ is the area measure on $\mathbb{C}$. Some simple computations with power series show that if $f(z)=\sum_{n \geq 0} a_{n} z^{n}$ is analytic in $U$, then

$$
\|f\|_{w}^{2}=\sum_{n \geq 0}\left|a_{n}\right|^{2} w_{n}
$$

where $w_{0}=1$ and for $n \geq 1$,

$$
w_{n}=2 \pi n^{2} \int_{0}^{1} r^{2 n-1} w(r) d r .
$$

It follows that $H_{w}$ is a separable Hilbert space of analytic functions in $U$ and that the polynomials are dense in $H_{w}$. Using the additional assumptions that $w$ is decreasing, concave, and satisfies $\lim _{r \rightarrow 1} w(r)=0$, locate the space $H_{w}$ between the well-known Dirichlet space $D$ obtained for $w=1$ and the Hardy space $H^{2}$ obtained for $w(r)=1-r, r \in[0,1)$; that is, $D \subset H_{w} \subseteq H^{2}$. The patterns for the above construction are the weighted Dirichlet spaces $D_{\alpha}$, $0<\alpha \leq 1$, corresponding to the choices $w_{\alpha}(r)=(1-r)^{\alpha}, r \in[0,1)$, and it is the aim of this paper to prove, in the more general context considered here, a result conjectured for the spaces $D_{\alpha}$ by S. Richter and A. Shields [3]; namely, the fact that every function in $H_{w}$ is the quotient of two bounded analytic

Received by the editors August 2, 1990 and, in revised form, November 16, 1990.

1980 Mathematics Subject Classification (1985 Revision). Primary 30H05; Secondary 47A15. 
functions in $H_{w}$. This result which is proved in $\S 2$, has some applications concerning the invariant subspaces of the multiplication operator defined on $H_{w}$ by

$$
\left(M_{z} f\right)(\zeta)=\zeta f(\zeta), \quad \zeta \in U, f \in H_{w} .
$$

Using (1.1) and (1.2) if follows easily that $M_{z}$ is a bounded weighted shift on $H_{w}$. From the result mentioned above it turns out that every nontrivial invariant subspace of $M_{z}$ contains a nontrivial bounded function, that each two nontrivial invariant subspaces have a nontrivial intersection and that each nontrivial invariant subspace has the codimension one property. For the usual Dirichlet space $D$, this was proved by S. Richter and A. Shields in [3]. These results provide positive answers to the corresponding questions for the spaces $D_{\alpha}[3$, Conjectures 1 and 2] and are proved in $\S 3$. The method used for the proofs also implies the following cyclicity theorem for the spaces $H_{w}$, related to Question 3 in [1]. A function whose modulus is greater or equal to the modulus of a cyclic vector for $M_{z}$ must also be a cyclic vector.

\section{BOUNDED FUNCTIONS IN $H_{w}$}

We begin with a general change of variable formula that is used in order to obtain an equivalent form of the norm on $H_{w}$.

2.1. Proposition. Let $\phi$ be a nonconstant analytic function in $U$ and $u, v$ be nonnegative measurable functions on $\mathbb{C}$ with respect to area measure. Then

$$
\int_{U}(u \circ \phi) v\left|\phi^{\prime}\right|^{2} d m=\int_{\phi(U)} u(\zeta)\left(\sum_{\phi(z)=\zeta} v(z)\right) d m(\zeta) .
$$

This result is actually known and was proved for $v(z)=-\log |z|$ in [4]. We include a sketch of its proof only for the sake of completeness.

Proof. There exists a sequence of pairwise disjoint sets $R_{n} \subset U, n \geq 1$, of the form $R_{n}=\left\{z \in U ; a_{n}<|z|<b_{n}, \alpha_{n}<\arg z<\beta_{n}\right\}$ such that $\phi_{\mid R_{n}}$ is injective and $m\left(U \backslash \bigcup_{n \geq 1} R_{n}\right)=0$. By the usual change of variable formula, we have

$$
\int_{U}(u \circ \phi) v\left|\phi^{\prime}\right|^{2} d m=\sum_{n \geq 1} \int_{\phi\left(R_{n}\right)} u\left[v \circ\left(\phi_{\mid R_{n}}\right)^{-1}\right] d m .
$$

Let $\chi_{n}$ be the characteristic function of $\phi\left(R_{n}\right)$ and $\phi_{n}$ be a measurable extension of $\left(\phi_{\mid R_{n}}\right)^{-1}$. We have

$$
\sum_{n \geq 1} \int_{\phi\left(R_{n}\right)} u\left(v \circ \phi_{n}\right) d m=\int_{\mathbb{C}} u\left[\sum_{n \geq 1} \chi_{n}\left(v \circ \phi_{n}\right)\right] d m .
$$

If $\Gamma$ is the complement of $\bigcup_{n \geq 1} R_{n}$, then $m(\phi(\Gamma))=0$ and for each $\zeta \in$ $\phi(U) \backslash \phi(\Gamma)$

$$
\sum_{n \geq 1} \chi_{n}(\zeta) v \circ \phi_{n}(\zeta)=\sum_{\phi(z)=\zeta} v(z)
$$

i.e. the above sums coincide a.e. on $\phi(U)$. 
2.2. Corollary. If $f \in H_{w}$ is nonconstant, then

$$
\|f\|_{w}^{2}=|f(0)|^{2}+\int_{f(U)}\left(\sum_{f(z)=\zeta} w(|z|)\right) d m(\zeta) .
$$

In what follows, for a nonconstant analytic function $f$ in $U, \zeta \in f(U)$ and $u$ a nonnegative measurable function on $[0,1)$, we denote

$$
N_{u, f}(\zeta)=\sum_{f(z)=\zeta} u(|z|) .
$$

In the special case when $u(r)=u_{0}(r)=\log 1 / r, r \in[0,1),(2.6)$ gives the usual Nevanlinna counting function of $f$ and we denote $N_{u_{0}, f}=N_{f}$

2.3. Lemma. Let $f \in H_{w}$ be nonconstant and for $z, \lambda \in U$ let $\varphi_{z}(\lambda)=(z+\lambda) \times$ $(1+\bar{z} \lambda)^{-1}$. Then for every $\zeta \in f(U)$

$$
N_{w, f}(\zeta)=-\frac{1}{2 \pi} \int_{U} \Delta \tilde{w}(z) N_{f \circ \varphi_{z}}(\zeta) d m(z),
$$

where $\tilde{w}$ is defined on $U$ by $\tilde{w}(z)=w(|z|)$ and $\Delta$ denotes the Laplace operator. Proof. Since $\lim _{|z| \rightarrow 1} \tilde{w}(z)=0$, by Green's theorem, for every $\lambda \in U$ we have

$$
\begin{aligned}
w(|\lambda|) & =\tilde{w}(\lambda)=\frac{1}{2 \pi} \int_{U} \Delta \tilde{w}(z) \log \left|\frac{z-\lambda}{1-\bar{\lambda} z}\right| d m(z) \\
& =\frac{1}{2 \pi} \int_{U} \Delta \tilde{w}(z) \log \left|\varphi_{z}^{-1}(\lambda)\right| d m(z) .
\end{aligned}
$$

Moreover, $\Delta \tilde{w}(z)=(1 /|z|) w^{\prime}(|z|)+w^{\prime \prime}(|z|) \leq 0$ because $w$ is decreasing and concave. Then by (2.8) and the monotone convergence theorem, we obtain

$$
\begin{aligned}
N_{w, f}(\zeta) & =\frac{1}{2 \pi} \sum_{f(\lambda)=\zeta} \int_{U} \Delta \tilde{w}(z) \log \left|\varphi_{z}^{-1}(\lambda)\right| d m(z) \\
& =-\frac{1}{2 \pi} \int_{U} \Delta \tilde{w}\left(\sum_{f(\lambda)=\zeta}-\log \left|\varphi_{z}^{-1}(\lambda)\right|\right) d m(z) .
\end{aligned}
$$

Finally for $\zeta \in f(U)$ and $z, \lambda \in U$, we have that $f(\lambda)=\zeta$ if and only if $f \circ \varphi_{z}\left(\varphi_{z}^{-1}(\lambda)\right)=\zeta$, which proves $(2.7)$.

From the fact that $H_{w}$ is contained in $H^{2}$, it follows that each function $f \in H_{w}$ has nontangential limits $f\left(e^{i \theta}\right)$ a.e. on $[0,2 \pi]$ and that its boundary function is in $L^{2}[0,2 \pi]$. For $z \in U$, let $P_{z}(\theta)=\operatorname{Re}\left(e^{i \theta}+z\right) /\left(e^{i \theta}-z\right)$, be the Poisson kernel. We prove

2.4. Proposition. Let $f \in H_{w}$. Then

$$
\|f\|_{w}^{2}=|f(0)|^{2}-\frac{1}{4} \int_{U} \Delta \tilde{w}(z)\left[\frac{1}{2 \pi} \int_{0}^{2 \pi} P_{z}(\theta)\left|f\left(e^{i \theta}\right)\right|^{2} d \theta-|f(z)|^{2}\right] d m(z) .
$$


Proof. For a nonconstant $f \in H_{w}$, we have

$$
\begin{aligned}
\|f\|_{w}^{2}-|f(0)|^{2} & =\int_{f(U)} N_{w, f} d m \\
& =\int_{f(U)}\left(-\frac{1}{2 \pi} \int_{U} \Delta \tilde{w}(z) N_{f \circ \varphi_{z}}(\zeta) d m(z)\right) d m(\zeta) \\
& =-\frac{1}{4} \int_{U} \Delta \tilde{w}(z)\left(\frac{2}{\pi} \int_{f(U)} N_{f \circ \varphi_{z}}(\zeta) d m(\zeta)\right) d m(z),
\end{aligned}
$$

by Corollary 2.2, Lemma 2.3, and Fubini's theorem. By (2.1) and the Littlewood-Paley formula

$$
\begin{aligned}
\frac{2}{\pi} \int_{f(U)} N_{f \circ \varphi_{z}} d m & =\frac{2}{\pi} \int_{U}\left|\left(f \circ \varphi_{z}\right)^{\prime}(\lambda)\right|^{2} \log \frac{1}{|\lambda|} d m \\
& =\left\|f \circ \varphi_{z}\right\|_{H^{2}}^{2}-\left|f \circ \varphi_{z}(0)\right|^{2} .
\end{aligned}
$$

Obviously $f \circ \varphi_{z}(0)=f(z)$ and by elementary computations with harmonic measures for the unit disc, we obtain

$$
\left\|f \circ \varphi_{z}\right\|_{H^{2}}^{2}=\frac{1}{2 \pi} \int_{0}^{2 \pi}\left|f \circ \varphi_{z}\left(e^{i \theta}\right)\right|^{2} d \theta=\frac{1}{2 \pi} \int_{0}^{2 \pi} P_{z}(\theta)\left|f\left(e^{i \theta}\right)\right|^{2} d \theta,
$$

and the proof is complete.

2.5. Corollary. Let $f \in H_{w}$ and $F$ be its outer factor. Then $F \in H_{w}$ and

$$
\|F\|_{w}^{2}-|F(0)|^{2} \leq\|f\|_{w}^{2}-|f(0)|^{2} .
$$

Proof. For every $z \in U$, we have $|f(z)| \leq|F(z)|$ and $\left|f\left(e^{i \theta}\right)\right|=\left|F\left(e^{i \theta}\right)\right|$ a.e. on $[0,2 \pi]$. Thus

$$
\frac{1}{2 \pi} \int_{0}^{2 \pi} P_{z}(\theta)\left|F\left(e^{i \theta}\right)\right|^{2} d \theta-|F(z)|^{2} \leq \frac{1}{2 \pi} \int_{0}^{2 \pi} P_{z}(\theta)\left|f\left(e^{i \theta}\right)\right|^{2} d \theta-|f(z)|^{2},
$$

and the result follows by Proposition 2.4.

For a function $f$ in the Nevanlinna class $N, f \neq 0$, we denote by $\phi_{f}$ the outer function satisfying $\left|\phi_{f}\left(e^{i \theta}\right)\right|=\min \left\{1,1 /\left|f\left(e^{i \theta}\right)\right|\right\}$ a.e. on $[0,2 \pi]$; that is,

$$
\phi_{f}(z)=\exp \frac{1}{2 \pi} \int_{0}^{2 \pi} \frac{e^{i \theta}+z}{e^{i \theta}-z} \log \min \left\{1,1 / \mid f\left(e^{i \theta} \mid\right\} d \theta .\right.
$$

Our main result is

2.6. Theorem. Let $f \in H_{w}, f \neq 0$. Then $\phi_{f}, f \phi_{f}$ are in $H_{w}$ and satisfy

$$
\left\|\phi_{f}\right\|_{w}^{2}-\left|\phi_{f}(0)\right|^{2} \leq\|f\|_{w}^{2}-|f(0)|^{2} \quad \text { and } \quad\left\|f \phi_{f}\right\|_{w} \leq\|f\|_{w} \text {. }
$$

The proof uses the following inequalities:

2.7. Lemma. Let $(X, \mu)$ be a probability space and $f \in L^{1}(\mu)$ such that $f>0$ $\mu$-a.e. on $X$ and $\log f \in L^{1}(\mu)$. Let

$$
E(f)=\int_{X} f d \mu-\exp \int_{X} \log f d \mu .
$$


Then

$$
E(\min \{1, f\}) \leq E(f)
$$

and

$$
E(\max \{1, f\}) \leq E(f) .
$$

Proof. Let $A=\{x \in X, f(x) \geq 1\}$ and assume that $\alpha=\mu(A)$ is in $(0,1)$, otherwise both inequalities are obviously satisfied. The inequality $(2.19)$ is equivalent to

$$
\int_{A}(f-1) d \mu \geq \exp \left(\int_{X \backslash A} \log f d \mu\right)\left[\exp \left(\int_{A} \log f d \mu\right)-1\right] .
$$

We have

$$
\begin{aligned}
& \exp \left(\int_{X \backslash A} \log f d \mu\right)\left[\exp \left(\int_{A} \log f d \mu\right)-1\right] \\
& \quad \leq\left(\exp \frac{1}{\alpha} \int_{A} \log f d \mu\right)^{\alpha}-1 \leq\left(\frac{1}{\alpha} \int_{A} f d \mu\right)^{\alpha}-1,
\end{aligned}
$$

by Jensen's inequality. Now it is easy to verify by differentiation that for every $t \geq 0$ and $0<\beta<1$,

$$
t-\beta \geq(t / \beta)^{\beta}-1,
$$

and (2.19) follows letting $t=\int_{A} f d \mu$ and $\beta=\alpha$.

The second inequality is very similar. Indeed, we have as above that $(2.20)$ is equivalent to

$$
\int_{X \backslash A}(f-1) d \mu \geq \exp \left(\int_{A} \log f d \mu\right)\left[\exp \left(\int_{X \backslash A} \log f d \mu\right)-1\right],
$$

and that the right side of $(2.24)$ is less or equal to

$$
\left(\exp \frac{1}{1-\alpha} \int_{X \backslash A} \log f d \mu\right)^{1-\alpha}-1 \leq\left(\frac{1}{1-\alpha} \int_{X \backslash A} f d \mu\right)^{1-\alpha}-1,
$$

by Jensen's inequality. Then (2.20) follows from (2.23) with $t=\int_{X \backslash A} f d \mu$ and $\beta=1-\alpha$.

Proof of Theorem 2.6. Let $f \in H_{w}, f \in I F$ with $I$ inner and $F$ outer. An application of (2.19) with $X=[0,2 \pi], d \mu=(1 / 2 \pi) P_{z} d \theta$, yields

$$
\begin{aligned}
& \frac{1}{2 \pi} \int_{0}^{2 \pi} P_{z}(\theta)\left|f \phi_{f}\left(e^{i \theta}\right)\right|^{2} d \theta-\left|f \phi_{f}(z)\right|^{2} \\
& \quad \leq \frac{1}{2 \pi} \int_{0}^{2 \pi} P_{z}(\theta)\left|F\left(e^{i \theta}\right)\right|^{2} d \theta-|I(z)|^{2}|F(z)|^{2} \\
& \quad=\frac{1}{2 \pi} \int_{0}^{2 \pi} P_{z}(\theta)\left|f\left(e^{i \theta}\right)\right|^{2} d \theta-|f(z)|^{2},
\end{aligned}
$$

and the second inequality in (2.17) follows by Proposition 2.3. Further, we have $\left|1 / \phi_{f}\left(e^{i \theta}\right)\right|=\max \left\{1,\left|f\left(e^{i \theta}\right)\right|\right\}$, a.e. on $[0,2 \pi]$ and for $z \in U$,

$$
\left|1 / \phi_{f}(z)\right|=\frac{1}{2 \pi} \int_{0}^{2 \pi} P_{z}(\theta) \log \max \left\{1,\left|f\left(e^{i \theta}\right)\right|^{2}\right\} d \theta .
$$


We apply (2.20) to obtain

$$
\begin{aligned}
& \frac{1}{2 \pi} \int_{0}^{2 \pi} P_{z}(\theta)\left|1 / \phi_{f}\left(e^{i \theta}\right)\right|^{2} d \theta-\left|1 / \phi_{f}(z)\right|^{2} \\
& \quad=E\left(\max \left\{1,|f|^{2}\right\}\right) \leq E\left(|f|^{2}\right) \\
& \quad \geq \frac{1}{2 \pi} \int_{0}^{2 \pi} P_{z}(\theta)\left|f\left(e^{i \theta}\right)\right|^{2} d \theta-|f(z)|^{2},
\end{aligned}
$$

for all $z \in U$. Thus by Proposition 2.3, $1 / \phi_{f} \in H_{w}$ and

$$
\left\|1 / \phi_{f}\right\|_{w}^{2}-\left|1 / \phi_{f}(0)\right|^{2} \leq\|f\|_{w}^{2}-|f(0)|^{2} .
$$

Finally, we have $\phi_{f}^{\prime}=-\phi_{f}^{2}\left(1 / \phi_{f}\right)^{\prime}$ and $\left|\phi_{f}\right| \leq 1$ in $U$, which proves the other inequality in (2.17).

Since $\left|\phi_{f}\right|,\left|f \phi_{f}\right| \leq 1$ in $U$ and $f=f \phi_{f} / \phi_{f}$, from Theorem 2.6 we obtain:

2.7. Corollary. Every function in $H_{w}$ is the quotient of two bounded functions in $H_{w}$.

\section{INVARIANT SUBSPACES}

The present section contains some applications of Theorem 2.6 concerning the invariant subspaces of the multiplication operator on $H_{w}$ defined by (1.4). A closed subspace $\mathscr{M}$ of $H_{w}$ is called invariant if $M_{z} \mathscr{M} \subset \mathscr{M}$. For a function $f \in H_{w}$ we denote by $[f]$ the smallest invariant subspace containing $f$; that is, the closure of the polynomial multiples of $f$ in $H_{w}$. In order to prove the main result of this section we use the same method as in [3]. Let $H^{\infty}$ be the algebra of bounded analytic functions in $U$ with the norm $\|g\|_{\infty}=\sup _{z \in U}|g(z)|$, $g \in H^{\infty}$. We have

3.1. Lemma. If $f \in H_{w}$ and $g \in H^{\infty}$ such that $g f \in H_{w}$, then $g f \in[f]$.

The proof of the lemma is based on some simple properties of the linear operators $T_{t}, 0 \leq t<1$, defined on the set $H(U)$ of analytic functions in $U$ by

$$
\left(T_{t} h\right)(z)=\frac{1}{1-t} \int_{t}^{1} h(s z) d s \quad z \in U, h \in H(U) .
$$

We obviously have $\lim _{t-1}\left(T_{t} h\right)(z)=h(z)$ for all $z \in U$ and $h \in H(U)$. Some other properties are summarized below. For $h \in H(U)$ and $t \in[0,1)$, we denote by $h_{t}$ the function given by $h_{t}(z)=h(t z), z \in U$.

3.2. Lemma. For every $t \in[0,1)$, we have

(i) $\left(M_{z} T_{t} h\right)^{\prime}=\frac{h-t h_{t}}{1-t}, h \in H(U)$.

(ii) If $h \in H_{w}$, then $T_{t} h \in H_{w}$ and $\left\|T_{t} h\right\|_{w} \leq\|h\|_{w}$.

(iii) If $h \in H^{\infty}$, then $T_{t} h \in H_{w}$ and $f \bar{T}_{t} h \in H_{w}$ whenever $f \in H_{w}$. Furthermore in this case, $f T_{t} h \in[f]$.

Proof. (i) For every $\zeta \in U$ and $h \in H(U)$,

$$
\left(M_{z} T_{t} h\right)(\zeta)=\frac{1}{1-t} \int_{t \zeta}^{\zeta} h(\lambda) d \lambda
$$


(ii) If $h(z)=\sum_{n \geq 0} a_{n} z^{n}, z \in U$, then

$$
\left(T_{t} h\right)(z)=\sum_{n \geq 0}\left(\frac{1-t^{n+1}}{\mathrm{i}-t}\right) \frac{a_{n}}{n+1} z^{n}, \quad z \in U
$$

which shows that $T_{t} h \in H_{w}$ whenever $h \in H_{w}$ and $\left\|T_{t} h\right\|_{w} \leq\|h\|_{w}$.

(iii) From (i) we obtain that if $h \in H^{\infty}$ then $T_{t} h$ and $\left(T_{t} h\right)^{\prime}$ are both in $H^{\infty}$, hence $T_{t} h \in H_{w}$ and is also a multiplier. Moreover, there exists a sequence of polynomials $\left\{p_{n}\right\}$ with $\sup _{n}\left\|p_{n}^{\prime}\right\|_{\infty}<\infty$, converging pointwise to $T_{t} h$ on $U$. It follows that $\sup _{n}\left\|p_{n} f\right\|_{w}<\infty$, and that at least a subsequence of $\left\{p_{n} f\right\}$ converges weakly in $H_{w}$. Also, its limit must be $f T_{t} h$ because the point evaluations are bounded linear functionals on $H_{w}$. Thus, $f T_{t} h \in[f]$.

Proof of Lemma 3.1. Let $f, g$ be as in the statement. For every $t \in[0,1)$ we have $f T_{t} g \in[f]$ by Lemma 3.2 and $\lim _{t \rightarrow 1}\left(f T_{t} g\right)(z)=f(z) g(z)$ for all $z \in U$. We are going to show that the norms $\left\|f T_{t} g\right\|_{w}$ remain bounded when $t$ tends to 1 . Note first that by the definition of $H_{w}$, it follows easily that the operator $M_{z}$ is injective and has closed range on $H_{w}$, hence there exists a positive constant $c$ such that $\left\|f T_{t} g\right\| \leq c\left\|M_{z} f T_{t} g\right\|_{w}, t \in[0,1)$. Further,

$$
\begin{aligned}
\left\|M_{z} f T_{t} g\right\|_{w}^{2} & \leq 2 \int_{U}\left|f\left(M_{z} T_{t} g\right)^{\prime}\right|^{2} w d m+2 \int_{U}\left|f^{\prime} M_{z} T_{t} g\right|^{2} w d m \\
& \leq 2 \int_{U}\left|f\left(M_{z} T_{t} g\right)^{\prime}\right|^{2} w d m+2\|g\|_{\infty}^{2}\|f\|_{w}^{2},
\end{aligned}
$$

and by Lemma 3.2(i),

$$
\begin{aligned}
\left|f\left(M_{z} T_{t} g\right)^{\prime}\right| & =\left|f \frac{g-t g_{t}}{1-t}\right| \leq\left|\frac{f g-t f_{t} g_{t}}{1-t}\right|+\left|g_{t} \frac{f-t f_{t}}{1-t}\right|+\left|f g_{t}\right| \\
& =\left|\left(M_{z} T_{t} f g\right)^{\prime}\right|+\left|g_{t}\left(M_{z} T_{t} f\right)^{\prime}\right|+\left|f g_{t}\right| .
\end{aligned}
$$

We obtain

$$
\begin{aligned}
\int_{U}\left|f\left(M_{z} T_{t} g\right)^{\prime}\right|^{2} w d m \leq & 3\left\|M_{z} T_{t} f g\right\|_{w}^{2}+3\|g\|_{\infty}^{2}\left\|M_{z} T_{t} f\right\|_{w}^{2} \\
& +3\|g\|_{\infty}^{2} \int_{U}|f|^{2} w d m .
\end{aligned}
$$

This leads to an estimation of the form

$$
\left\|f T_{t} g\right\|_{w}^{2} \leq c_{1}\|f g\|_{w}^{2}+c_{2}\|g\|_{\infty}^{2}\|f\|_{w}^{2},
$$

where $c_{1}, c_{2}$ are positive constants independent of $t$. As in the proof of Lemma 3.2(iii), there exists a sequence $\left\{t_{n}\right\}$ in $[0,1)$, tending to 1 such that $f T_{t_{n}} g$ tends weakly to $f g$ in $H_{w}$, hence $g f \in[f]$.

A function $f \in H_{w}$ is called a cyclic vector for $M_{z}$ if $[f]=H_{w}$. From Lemma 3.1 we obtain

3.3. Corollary. If $f, g \in H_{w}, g$ is a cyclic vector for $M_{z}$ and $|f(z)| \geq|g(z)|$ for all $z \in U$, then $f$ is also cyclic.

Proof. If $h=g / f$, then $h \in H^{\infty}$ and $h f=g$, i.e. $h f \in H_{w}$. Then by Lemma 3.1, $g \in[f]$, which shows that $f$ is cyclic.

Remark. The above result remains true if $H_{w}$ is replaced by the Dirichlet space $D$, because Lemma 3.1 holds for this space as well, with the same proof. This 
problem was raised for general Banach spaces of analytic functions by L. Brown and A. Shields [1, Question 3].

The main result of this section is

3.4. Theorem. Let $\mathscr{M} \neq\{0\}, \mathscr{N} \neq\{0\}$ be invariant subspaces for the operator $M_{z}$ on $H_{w}$. Then (i) $\mathscr{M} \cap H^{\infty} \neq\{0\}$ and (ii) $\mathscr{M} \cap \mathscr{N} \neq\{0\}$.

Proof. (i) Let $f \in \mathscr{M}, f \neq 0$. By Theorem 2.6 there exist functions $g$, $h \in H^{\infty} \cap H_{w}$ such that $f=g / h$ and, by Lemma 3.1, $g=h f \in[f] \subset \mathscr{M}$. (ii) If $g \in \mathscr{M}, h \in \mathscr{N}, g, h \neq 0$ are bounded then $g h \in[g] \cap[h] \subset \mathscr{M} \cap \mathcal{N}$.

Theorem 3.4(ii) states that the operator $M_{z}$ on $H_{w}$ is cellular indecomposable and answers affirmatively Conjecture 1 of [3]. As it was pointed out in [3] this implies the fact that each nontrivial invariant subspace $\mathscr{M}$ of $M_{z}$ has the codimension one property that is, $(z-\lambda) \mathscr{M}$ is a closed subspace of $\mathscr{M}$ having codimension 1 in $\mathscr{M}$, for every $\lambda \in U$. This follows from results obtained by S. Richter in [2] and Theorem 3.4. Indeed, if $\mathscr{M}$ is such a subspace and $f$, $g \in \mathscr{M} \backslash\{0\}$, then $[f] \cap[g] \neq\{0\}$ by Theorem 3.3 and by [2, Corollaries 3.12 and 3.15] $\mathscr{M}$ has the codimension one property.

\section{ACKNOWLEDGMENT}

The author is greatly in debted to the referee who simplified the proof of the main result and pointed out an error in the first version of this paper.

\section{REFERENCES}

1. L. Brown and A. Shields, Cyclic vectors in the Dirichlet space, Trans. Amer. Math. Soc. 285 (1984), 269-304.

2. S. Richter, Invariant subspaces in Banach spaces of analytic functions, Trans. Amer. Math. Soc. 304 (1987), 585-616.

3. S. Richter and A. Shields, Bounded analytic functions in the Dirichlet space, Math. Z. 198 (1988), 151-159.

4. J. Shapiro, The essential norm of composition operator, Ann. of Math. (2) 125 (1987), 375-404.

Fachbereich Mathematik und Informatik, Fernuniversität Hagen, Postfach 940, 5800 HAGEN 1, West GeRMANY 